\title{
SYNTHESIS AND CHARACTERIZATION OF SOME NOVEL CHALCONE DERIVATIVES
}

\author{
A. Kishore Babu ${ }^{1,2}$ and K. Selvaraju, ${ }^{2, *}$ \\ ${ }^{1}$ Research Scholar, Research and Development Centre, Bharathiar University, \\ Coimbatore-641 046, Tamil Nadu, India \\ 2,* Department of Chemistry, Sri Sairam Engineering College, \\ Chennai-600 044, Tamil Nadu,India. \\ *E-mail: jkselvaraju@gmail.com
}

\begin{abstract}
Chalcones are the important constituent of many natural sources and have a wide variety of biological activities. Following Claisen-Schmidt condensation reaction, a number of chalcones were prepared by the reaction between derivatives of acetophenones with variously substituted benzaldehydes in Sodium hydroxide solution and Ethanol medium at $25-30^{\circ} \mathrm{C}$. The synthesized chalcones were confirmed by Infrared, $\mathrm{H}^{1} \mathrm{NMR}$ and Mass studies. The above chalcones were checked for their antibacterial and antifungal activities.
\end{abstract}

Keywords:Chalcone,Claisen-Schmidt condensation, Benzaldehyde, Acetophenone, Antibacterial activity, Antifungal activity.

(C) RASĀYAN. All rights reserved

\section{INTRODUCTION}

Due to the rapid development of resistance towards antibiotics, the need for the development of new antibacterial agents has been a very important step for research. The leading challenge for human life are diverse diseases and the fast development of microbial resistance towards existing drugs. Researchers nowadays are directed towards the design of new drugs with better pharmacokinetic profile and lesser toxicity.

Chalcones and its derivatives form an important group of natural products. Chalcones act as a well known key intermediate for various heterocyclic compounds. Chalcone derivatives have exhibited various biological activities such as antimicrobial ${ }^{1,2}$, antitumour ${ }^{3,4}$ anti-inflammatory ${ }^{5}$, antibacterial ${ }^{6,7}$, antimalarial $^{8}$, antioxidant ${ }^{9,10}$, antitoxicity ${ }^{10}$ and anticancer ${ }^{6,11}$.Chalcones were also synthesized by Microwave assistance ${ }^{12}$ and proved to give better yield. In present studies, we synthesized and characterized five novel chalcone derivatives(5a-e) derived from variously substituted acetophenones with differently substituted benzaldehydes.

\section{EXPERIMENTAL}

Synthesis of $N$-(3-acetyl phenyl)-2-chloroacetamide (3)

To a stirred solution of 3 -amino acetophenone in glacial acetic acid at $25-30^{\circ} \mathrm{C}$, chloroacetylchloride in acetic acid was added. After completion of the reaction, 0.5 M Sodium acetate solution was charged and stirred for 30 minutes. The obtained product was filtered and dried at $55-60^{\circ} \mathrm{C}$ for 10 hours in a hot air oven. The crude product was further purified using ethanol.

Synthesis of 2-(4-acetamidophenoxy)- $N$-(3-acetyl-phenyl)acetamide (4)

To a stirred solution of Potassium carbonate in $N, N$-dimethylformamide ,a solution of $N$-(3-Acetylphenyl)-2-chloro-acetamide (3) in $N, N$-dimethylformamide was charged slowly at $25-30^{\circ} \mathrm{C}$ and maintained for 30 min after that $\mathrm{N}$-(Hydroxyphenyl)acetamide (4) was added and heated for 3 hours at reflux temperature.

Rasayan J. Chem., 11(4), 1501-1505(2018)

http://dx.doi.org/10.31788/RJC.2018.1144037

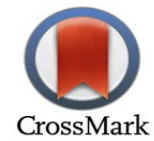


The reaction progress was monitored by TLC, once the reaction was complete, it was then poured into chilled water. The obtained product was filtered, washed with water, dried in a hot air oven and weighed. The crude product was further purified using rectified spirit.

\section{General Procedure for the Synthesis of Substituted chalcones (5 a-d)}

An equimolar quantity of 2-(4-Acetamidophenoxy)- $N$-(3-Acetyl-phenyl)acetamide and substituted benzaldehyde were taken in ethanol. $20 \% \mathrm{NaOH}$ solution was added and the reaction mass was continued for 24 hours. After the reaction was complete, it was then quenched into water and acidified with $10 \%$ Hydrochloric acid and the obtained product was filtered, dried in a hot air oven and weighed. The crude product was further purified using ethanol.

\section{General Procedure for the Synthesis of Substituted chalcones $(5 \mathrm{e})$}

Equimolar quantity of 2-(4-Acetamidophenoxy)- $N$-(3-Acetyl-phenyl)acetamide and Furfuraldehyde were taken in ethanol. $20 \% \mathrm{NaOH}$ solution was added and the reaction mass was maintained for 24 hours. After the reaction was complete, it is then quenched into chilled water and acidified with $10 \%$ Hydrochloric acid to obtain the product. The crude product was further purified using ethanol.

\section{N-(3-acteylphenyl)-2-chloroacetamide (3)}

Yellowish brown solid, ${ }^{1} \mathrm{H}$ NMR (300MHz DMSO): $\delta 2.61(\mathrm{~s}, 3 \mathrm{H}), 4.21(\mathrm{~s}, 2 \mathrm{H}), 7.44(\mathrm{t}, 1 \mathrm{H}, J=7.8 \mathrm{~Hz}$ ),7.74 (d, 1H, J=7.8Hz ), 7.90 (d, 1H, J=7.8Hz),8.05 (s, 1H,), 8.48 (s, 1H, NH).

\section{2-(4-acetamidophenoxy)-N-(3-acetylphenyl)acetamide (4)}

Yellowish brown solid, ${ }^{1} \mathrm{H}$ NMR (300MHz DMSO): $\delta 2.11(\mathrm{~s}, 3 \mathrm{H}), 2.60(\mathrm{~s}, 3 \mathrm{H}), 4.60(\mathrm{~s}, 2 \mathrm{H}), 6.93(\mathrm{~d}, 2 \mathrm{H}$, $J=8.1 \mathrm{HZ}), 7.41(\mathrm{t}, 1 \mathrm{H}, J=7.8 \mathrm{~Hz}), 7.52(\mathrm{~d}, 2 \mathrm{H}, J=8.1 \mathrm{~Hz}), 7.68(\mathrm{~d}, 1 \mathrm{H}, J=7.8 \mathrm{~Hz}), 7.96(\mathrm{~d}, 1 \mathrm{H}, J=7.8 \mathrm{~Hz})$, $8.21(\mathrm{~s}, 1 \mathrm{H}), 9.36(\mathrm{~s}, 1 \mathrm{H}), 9.43(\mathrm{~s}, 1 \mathrm{H})$.

\section{(E)-2-(4-acetamidophenoxy)-N-(3-(3-(4-dimethylamino)phenyl)acryloyl)phenyl)acetamide (5a)}

Yellowish brown solid, IR (KBr) $\left(\mathrm{cm}^{-1}\right)$ 3625(amide NH), $2672(\mathrm{CH}), 1067(\mathrm{C}=\mathrm{C}), 1697(\mathrm{CO}), 1519$ (amide CO), $2360(\mathrm{C}-\mathrm{N}) .{ }^{1} \mathrm{H}$ NMR : $\delta 2.03$ (s, 3H), 3.04 (s, 6H), 4.62 (s, 2H), 6.69 (d, 1H, J=7.8Hz), 6.93 $(\mathrm{d}, 2 \mathrm{H}, J=8.4 \mathrm{~Hz}), 7.38-7.43(\mathrm{~m}, 2 \mathrm{H}), 7.49(\mathrm{~d}, 2 \mathrm{H}, J=8.4 \mathrm{~Hz}), 7.57(\mathrm{~d}, 1 \mathrm{H}, J=7.8 \mathrm{~Hz}), 7.64-7.76(\mathrm{~m}, 3 \mathrm{H})$, $7.94-8.03(\mathrm{~m}, 2 \mathrm{H}), 8.25(\mathrm{~d}, 1 \mathrm{H}, J=7.8 \mathrm{~Hz}), 9.69(\mathrm{~s}, 1 \mathrm{H}), 10.08(\mathrm{~s}, 1 \mathrm{H})$. Mass of $\mathrm{C}_{27} \mathrm{H}_{27} \mathrm{~N}_{3} \mathrm{O}_{4}=457.20$.

\section{(E)-2-(4-acetamidophenoxy)-N-(3-(3-(p-tolyl)acryloyl)phenyl)acetamide(5b)}

White solid, IR (KBr) $\left(\mathrm{cm}^{-1}\right)$ 3502(amide $\left.\mathrm{NH}\right), 2823(\mathrm{CH}), 1168(\mathrm{C}=\mathrm{C}), 1658(\mathrm{CO}), 1608$ (amide CO), 2360(C-N). ${ }^{1} \mathrm{H}$ NMR : $\delta 2.05$ (s, 3H), 2.41 (s, 3H), 4.70 (, 2H), 6.98 (d, 2H, J=6.9Hz), 7.29 (d, 2H, $J=7.2 \mathrm{~Hz}), 7.53(\mathrm{~d}, 2 \mathrm{H}, J=7.5 \mathrm{~Hz}), 7.74-7.82(\mathrm{~m}, 4 \mathrm{H}), 7.89(\mathrm{~d}, 1 \mathrm{H}, J=6.6 \mathrm{~Hz}), 8.03(\mathrm{~d}, 1 \mathrm{H}, J=8.1 \mathrm{~Hz}), 8.26$ $(\mathrm{d}, 2 \mathrm{H}, J=7.8 \mathrm{~Hz}), 9.82(\mathrm{~s}, 1 \mathrm{H}), 10.27(\mathrm{~s}, 1 \mathrm{H})$. Mass of $\mathrm{C}_{26} \mathrm{H}_{24} \mathrm{~N}_{2} \mathrm{O}_{4}=428.17$.

\section{(E)-2-(4-acetamidophenoxy)-N-(3-(3-(o-tolyl)acryloyl)phenyl)acetamide(5c)}

Yellowish brown solid, IR (KBr) $\left(\mathrm{cm}^{-1}\right)$ 3494(amide NH), $2669(\mathrm{CH}), 1689$ (CO), 1608 (amide CO), $2360(\mathrm{C}-\mathrm{N}) .{ }^{1} \mathrm{H}$ NMR : $\delta 2.05(\mathrm{~s}, 3 \mathrm{H}), 2.56(\mathrm{~s}, 3 \mathrm{H}), 4.71(\mathrm{~s}, 2 \mathrm{H}), 6.98(\mathrm{~d}, 2 \mathrm{H}, J=8.1 \mathrm{~Hz}), 7.33(\mathrm{t}, 2 \mathrm{H}$, $J=9.0 \mathrm{~Hz}), 7.54-7.56(\mathrm{~m}, 2 \mathrm{H}), 7.72(\mathrm{~d}, 2 \mathrm{H}, \mathrm{J}=15.3 \mathrm{~Hz}), 7.93-7.90(\mathrm{~m}, 2 \mathrm{H}), 7.96-8.06(\mathrm{~m}, 2 \mathrm{H}), 8.29-8.38$ $(\mathrm{m}, 2 \mathrm{H}), 9.83(\mathrm{~s}, 1 \mathrm{H}), 10.31(\mathrm{~s}, 1 \mathrm{H})$. Mass of $\mathrm{C}_{26} \mathrm{H}_{24} \mathrm{~N}_{2} \mathrm{O}_{4}=428.17$.

2-(4-acetamidophenoxy)-N-(3-cinnamoylphenyl)acetamide(5d)Brown solid, IR $(\mathrm{KBr}) \quad\left(\mathrm{cm}^{-1}\right)$ 3490(amide NH), $2669(\mathrm{CH}), 1662(\mathrm{CO}), 1608$ (amide CO), 2360(C-N). ${ }^{1} \mathrm{H}$ NMR : $\delta 2.07$ (s, 3H), 4.60 (s, $2 \mathrm{H}), 6.92(\mathrm{~d}, 2 \mathrm{H}, J=8.4 \mathrm{~Hz}), 7.41-7.45(\mathrm{~m}, 2 \mathrm{H}), 7.50-7.53(\mathrm{~m}, 3 \mathrm{H}), 7.59-7.66(\mathrm{~m}, 3 \mathrm{H}), 7.73-7.76(\mathrm{~m}, 3 \mathrm{H})$, $7.98(\mathrm{~d}, 1 \mathrm{H}, J=8.7 \mathrm{~Hz}), 8.03(\mathrm{~s}, 1 \mathrm{H}), 9.46(\mathrm{~s}, 1 \mathrm{H}), 9.68(\mathrm{~s}, 1 \mathrm{H})$. Mass of $\mathrm{C}_{25} \mathrm{H}_{22} \mathrm{~N}_{2} \mathrm{O}_{4}=414.16$.

\section{(E)-2-(4-acetamidophenoxy)-N-(3-(3-(furan-2-yl)acryloyl)phenyl)acetamide (5e)}

Yellowish brown solid, IR (KBr) $\left(\mathrm{cm}^{-1}\right)$ 3491(amide NH), $2850(\mathrm{CH}), 1658(\mathrm{CO}), 1608$ (amide CO), 2360 (C-N). ${ }^{1} \mathrm{H}$ NMR : ${ }^{1} \mathrm{H}$ NMR (300MHz DMSO): $\delta 2.05$ (s, 3H), 4.65 (s, 2H), 6.60 ( s, 1H), 6.93 (d, $2 \mathrm{H}, J=9 \mathrm{~Hz}), 7.42-7.60(\mathrm{~m}, 6 \mathrm{H}), 7.74(\mathrm{~s}, 1 \mathrm{H}), 8.00-8.12(\mathrm{~m}, 2 \mathrm{H}), 8.35$ (s, 1H), $9.72(\mathrm{~s}, 1 \mathrm{H}), 10.17(\mathrm{~s}, 1 \mathrm{H})$. 
RASĀYAN J. Chem.

Vol. 11 | No. 4 |1501 - 1505| October - December | 2018

\section{RESULTS AND DISCUSSION}

The structure of Chalcone derivatives was confirmed by IR, $\mathrm{H}^{1} \mathrm{NMR}$ and Mass spectra. The various reactions are explained in Scheme-1.The chemical report of the chalcone derivatives is given in Table- 1 . $\mathrm{H}^{1}$ NMR Spectrum of compound $5 \mathrm{~b}$ is given in Fig.-1.

Table-1: Physical Constants of the Chalcone Derivatives

\begin{tabular}{c|c|c|c|c|c}
\hline $\begin{array}{c}\text { Comp. } \\
\text { Code }\end{array}$ & $\mathrm{R}$ & $\begin{array}{c}\text { Molecular } \\
\text { Formula }\end{array}$ & M. Wt & Yield \% & MP $\left({ }^{0} \mathrm{C}\right)$ \\
\hline $5 \mathrm{a}$ & $4-\left(\mathrm{CH}_{3}\right)_{3} \mathrm{~N}$ & $\mathrm{C}_{27} \mathrm{H}_{27} \mathrm{~N}_{3} \mathrm{O}_{4}$ & 457.52 & 45 & 90 \\
\hline $5 \mathrm{~b}$ & $4-\mathrm{CH}_{3}$ & $\mathrm{C}_{26} \mathrm{H}_{24} \mathrm{~N}_{2} \mathrm{O}_{4}$ & 428.48 & 65 & 110 \\
\hline $5 \mathrm{c}$ & $2-\mathrm{CH}_{3}$ & $\mathrm{C}_{26} \mathrm{H}_{24} \mathrm{~N}_{2} \mathrm{O}_{4}$ & 428.48 & 60 & 118 \\
\hline $5 \mathrm{~d}$ & $4-\mathrm{H}$ & $\mathrm{C}_{25} \mathrm{H}_{22} \mathrm{~N}_{2} \mathrm{O}_{4}$ & 414.45 & 50 & 142 \\
\hline $5 \mathrm{e}$ & $\mathrm{C}_{5} \mathrm{H}_{5} \mathrm{O}_{2}$ & $\mathrm{C}_{23} \mathrm{H}_{20} \mathrm{~N}_{2} \mathrm{O}_{5}$ & 404.42 & 60 & 125 \\
\hline
\end{tabular}

\section{Antibacterial Activity}

The synthesized chalcones were subjected to antibacterial studies. The compound $5 \mathrm{e}$ exhibited very good activity whereas the compounds $5 \mathrm{a}, 5 \mathrm{~b}, 5 \mathrm{c} \& 5 \mathrm{~d}$ exhibited good to reasonable activity against $E$. coli, S. aureus, K. pneumonia and B. subtilis at both the concentrations i.e. $100 \mu \mathrm{g} / \mathrm{ml}$ and $200 \mu \mathrm{g} / \mathrm{ml}$.

Antibacterial activity of all the compounds was carried out by disc diffusion technique. Anti-microbial activity was also tested in vitro against $E$. coli, S. aureus, K. pneumonia ,B. subtilis and referred with Streptomycin $(10 \mu \mathrm{g})$-standard drug. The inhibition Zones for the chalcones against organisms were calculated.

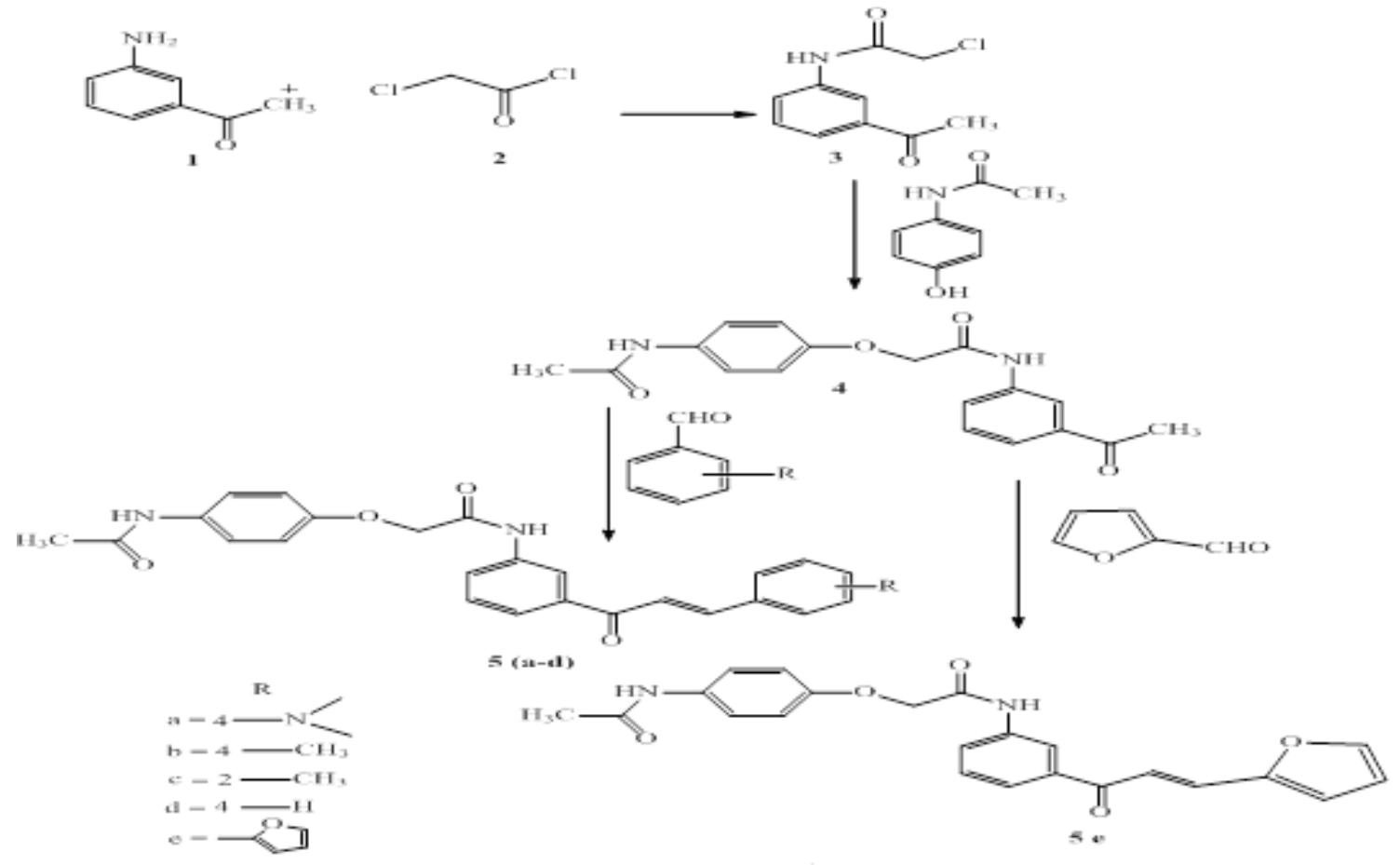

Disc-diffusion Assay

Scheme-1

The effect of various compounds on selected bacterial strains was assayed by Disc diffusion method. The concentrations of the test compounds were $100 \mu \mathrm{g}, 200 \mu \mathrm{g}$ and standard drug Streptomycin $10 \mu \mathrm{g} / \mathrm{disc}$. The Details of the organisms and their anti-microbial activity expressed in millimeters is given in Table-2.

\section{Antifungal Activity}

The synthesized chalcones were screened for Antifungal activity using Fluconazole $15 \mu \mathrm{g} \&$ Clotrimazole $15 \mu \mathrm{g}$ as Standard. The diameters of zone of inhibition observed were measured (Table-3). 
RASĀYAN $J$. Chem.

Vol. 11 | No. 4 |1501 - 1505| October - December | 2018

Antifungal activity of all the synthesized compounds was screened using Fluconazole $15 \mu \mathrm{g} \&$ Clotrimazole $15 \mu \mathrm{g}$ as a Standard drug. Anti-fungal activity was tested in vitro against C.albicans and A.niger, compounds 5a,5c and 5d showed good activity against both C.albicans and A.niger whereas the compounds $5 \mathrm{a}$ and $5 \mathrm{e}$ showed moderate activity against both the organisms.

Table-2: Antibacterial Activity

\begin{tabular}{c|c|c|c|c|c|c|c|c}
\hline \multirow{2}{*}{ Compound } & \multicolumn{9}{|c|}{ Zone of Inhibition (mm) } \\
\cline { 2 - 9 } & \multicolumn{2}{|c|}{ S. aereus } & \multicolumn{2}{c}{ B. subtilis } & \multicolumn{2}{c}{ E. coli } & \multicolumn{2}{c}{ K. pneumonia } \\
\cline { 2 - 9 } & $100 \mu \mathrm{g}$ & $200 \mu \mathrm{g}$ & $100 \mu \mathrm{g}$ & $200 \mu \mathrm{g}$ & $100 \mu \mathrm{g}$ & $200 \mu \mathrm{g}$ & $100 \mu \mathrm{g}$ & $200 \mu \mathrm{g}$ \\
\hline 5a & - & - & 10 & 14 & 9 & 15 & - & - \\
\hline $5 \mathrm{~b}$ & 9 & 13 & 8 & 12 & 10 & 14 & 7 & 9 \\
\hline $5 \mathrm{c}$ & - & 12 & - & 11 & - & 9 & - & - \\
\hline $5 \mathrm{~d}$ & 9 & 14 & - & 14 & 11 & 13 & - & 12 \\
\hline 5e & 10 & 15 & 10 & 13 & 9 & 14 & - & - \\
\hline $\begin{array}{c}\text { Streptomycin } \\
(10 \mu \mathrm{g})\end{array}$ & 24 & 23 & 25 & 17 & & & & \\
\hline
\end{tabular}

-Not active

Table-3: Antifungal Activity

\begin{tabular}{c|c|c|c|c}
\hline \multirow{2}{*}{ Compound } & \multicolumn{3}{|c}{ Zone of Inhibition (mm) } \\
\cline { 2 - 5 } & \multicolumn{2}{|c|}{ C.albicans } & \multicolumn{2}{c}{ A.niger } \\
\cline { 2 - 5 } & $100 \mu \mathrm{g}$ & $200 \mu \mathrm{g}$ & $100 \mu \mathrm{g}$ & $200 \mu \mathrm{g}$ \\
\hline $5 \mathrm{a}$ & 13 & 15 & 9 & 13 \\
\hline $5 \mathrm{~b}$ & 9 & 14 & 10 & 12 \\
\hline $5 \mathrm{c}$ & 12 & 16 & 11 & 13 \\
\hline $5 \mathrm{~d}$ & 14 & 18 & 8 & 12 \\
\hline $5 \mathrm{e}$ & 11 & 14 & 12 & 14 \\
\hline Fluconazole $(15 \mu \mathrm{g})$ & \multicolumn{2}{|c|}{-} & \multicolumn{2}{|c}{27} \\
\hline Clotrimazole $(15 \mu \mathrm{g})$ & \multicolumn{2}{|c|}{22} & \multicolumn{2}{c}{-} \\
\hline
\end{tabular}

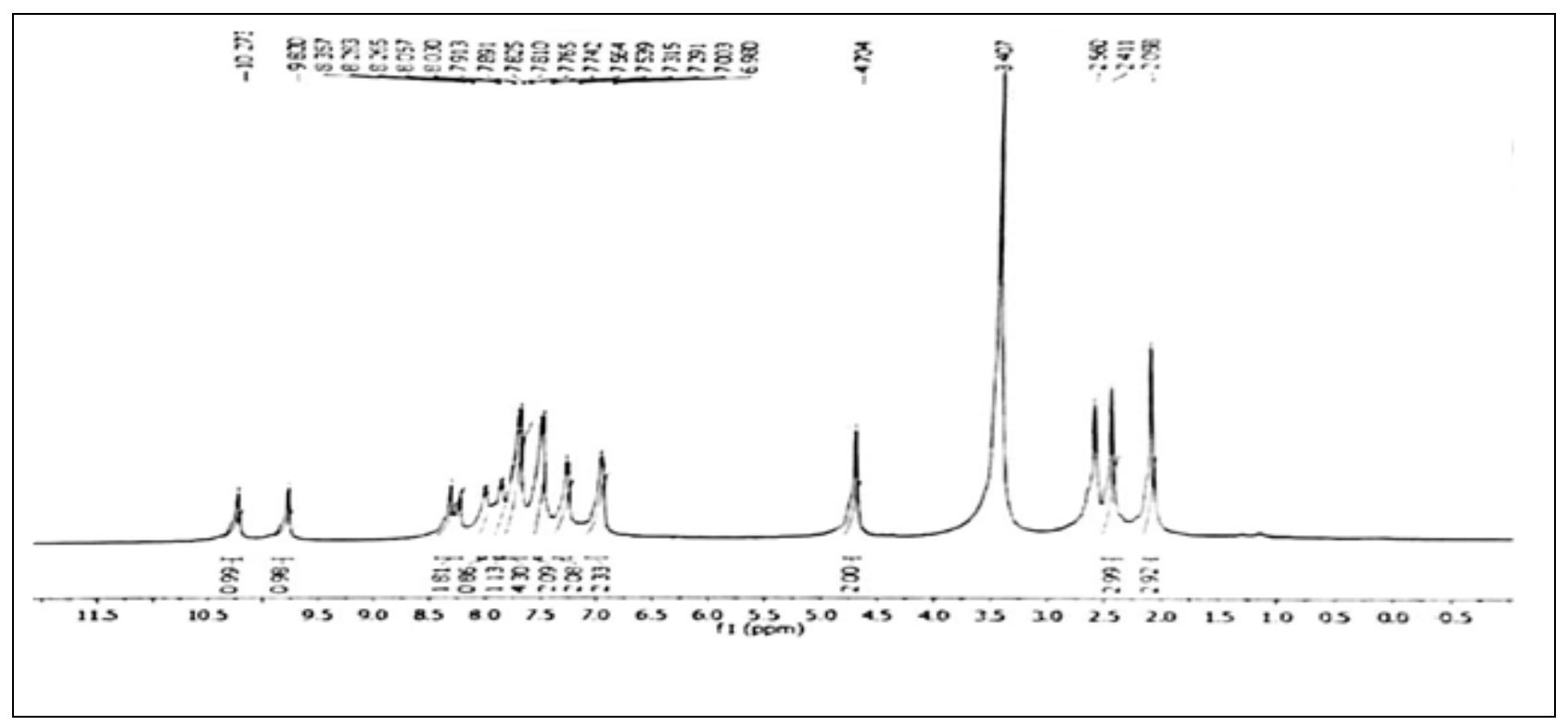

Fig.-1:H $\mathrm{H}^{1} \mathrm{NMR}$ Spectrum of compound $5 \mathrm{~b}$

CONCLUSION

The current study reveals the synthesis of some novel substituted chalcone derivatives. Chalconeswere confirmed by Infrared, $\mathrm{H}^{1} \mathrm{NMR}$ and Mass studies. Chalcones were evaluated for Antibacterial and Antifungal activities and were found to exhibit good to reasonable activity. 
RASĀYAN J. Chem.

Vol. 11 | No. 4 |1501 - 1505| October - December | 2018

\section{ACKNOWLEDGMENT}

Authors are thankful to Cephas Medical Pvt Ltd, B13, MEPZ Special Economic Zone (SEZ), Chennai, India for providing the facilities to carry out our research work.

\section{REFERENCES}

1. Y. Rajendra Prasad, A. Lakshmana Rao and R. Rambabu, E.J. Chem, 5(3),461 (2008), DOI: $10.1155 / 2008 / 876257$

2. Bhagyesh Baviskar, Sureshbhi Patel, Bhushan Baviskar, S. S. Khadabadi, Mahendra Shiradkar, Asian J. Res.Chem.,1(2), 67 (2008).

3. S. Shibata, Stem Cells, 12(1), 44 (1994), DOI:10.1002/stem.5530120109.

4. S. Cassia, S.P. Mizuno, S. Nanjoo, M.R. Agnes, Bio . org Med Chem. Lett 20, 7385 (2010), DOI: 91.1211/ijcpa.v2i3.633

5. V. Susanne, B. Matej, J. Guido. Eur J. Med. Chem., 45, 2206 (2010), DOI: 10.1016/j.ejmech.2010.01.060

6. MHM Mumtaz,BK Ishwar, BC Revanasiddappa, S Abubaker, D.R. Bharathi, Pharmacologyonline,3, 880 (2011).

7. X.L. Liu, Y.J. Xu, M.L. Go,.Eur. J. Med. Chem, 43(8), 1681 (2008), DOI:10.1016/j.ejmech.2007.10.007

8. M. Liu, P. Wilarirat, M.L. Go,.J. Med.Chem.,44(25), 4443 (2001), DOI: 10.1021/jm0101747

9. D.P. Belsare, S.C. Pal, A.A. Kazi, R.S. Kankate, S.S. Vanjari, Inter. J. ChemTech Res., 2(2),1080 (2010).

10. Mohd. Rayees Ahmed, V.Girija Sastry, Nasreen Bano, S. Ravichandra and M. Raghavendra Rasayan J.Chem, 4(2), 289 (2011).

11. Anjani Solankee, Yogesh Prajapati, Rasayan J.Chem, 2(1), 9 (2009).

12. Y.K.Srivastava, Rasayan J.Chem., 1(4), 884 (2008).

[RJC-4037/2018] 